\title{
AVALIAÇÃO DO CONTROLE POSTURAL E DA PRESSÃO PLANTAR EM PESSOAS COM ALTERAÇÃO VISUAL
}

José Carlos de Lima Júnior ${ }^{1}$, Débora Cristina Nunes Carvalho ${ }^{1}$, Rafael Victor Ferreira do Bonfim ${ }^{2}$, Vivaldo Xavier Silva Sousa ${ }^{3}$

${ }^{1}$ Centro de Ensino Unificado de Teresina - CEUT. ${ }^{2}$ Faculdade Integral Diferencial - FACID/DeVry. ${ }^{3}$ Associação de Ensino Superior do Piauí (Aespi). E-mail: lima juniorviana@hotmail.com

\section{RESUMO}

O estudo objetivou avaliar o controle postural e a pressão plantar em pessoas com alteração visual, utilizando como referência o estrabismo. Participaram do estudo 34 voluntários divididos em dois grupos: Grupo com Alteração Visual (GAV) com 17 indivíduos com estrabismo e um Grupo Controle (GC) formado por 17 indivíduos com visão normal. Todos os participantes foram submetidos às avaliações da pressão plantar e do equilíbrio por meio do exame da baropodometria computadorizada. Não houve diferença significativa na distribuição da pressão na região plantar entre os dois grupos. Na avaliação do equilíbrio, a velocidade média de oscilação e o deslocamento látero-lateral, com olhos abertos, apresentaram diferença estatística entre os grupos, bem como o deslocamento ântero-posterior, com olhos fechados. Conclui-se que não houve diferença estatisticamente significante na distribuição da pressão plantar ao comparar os dois grupos. Há alterações significativas no equilíbrio em indivíduos com alteração visual no deslocamento L/L e na velocidade média de oscilação, quando com os olhos abertos, no entanto existe uma correlação positiva em relação ao deslocamento $A / P$ na condição olhos fechados, quando comparados com os indivíduos com visão normal.

Palavras-chave: equilíbrio postural, visão ocular, transtornos visuais, estrabismo, deformidades do pé.

\section{POSTURAL CONTROL EVALUATION AND PLANT PRESSURE IN PEOPLE WITH VISUAL CHANGE}

\begin{abstract}
The study aimed to evaluate the postural control and plant pressure in people with visual change, using as reference the strabismus. Participated in the study 34 volunteers divided into two groups: Group with Visual Amendment (GAV) with 17 individuals with strabismus and a Control Group (CG) consisted of 17 subjects with normal vision. All participants were subjected to pressure ratings planting and balance by examining the baropodometry. There was no significant difference in the distribution of pressure in the plantar region between the two groups. In the assessment of the balance, the average speed of oscillation and the side-to-side scrolling, with open eyes, showed statistical differences between groups, and the anteroposterior displacement, with eyes closed. It was concluded that there was no statistically significant difference in plantar pressure distribution when comparing the two groups. There are significant changes in the balance in individuals with visual change in the $L / L$ displacement and average speed of oscillation, when with open eyes, however there is a positive correlation with respect to the displacement $A / P$ in the eyes closed condition, compared to subjects with normal vision.

Keywords: postural balance, eye vision, visual disorders, strabismus, foot deformities.
\end{abstract}

\section{INTRODUÇÃO}

A manutenção do equilíbrio corporal deve-se a integração de várias estruturas, que compõem um sistema de comando chamado de controle postural ${ }^{1}$.
O equilíbrio é um complexo mecanismo sensório-motor e base para todo movimento ${ }^{2}$, onde sua função dependente da integridade anatômica e funcional do sistema visual, bem como da correlação entre os sistemas vestibular e proprioceptivo ${ }^{3,4}$. 
As informações provenientes destes sistemas sensoriais são integradas e processadas no sistema nervoso central, o qual se encarrega de planejar e executar as ações motores pertinentes à manutenção ou à restauração do equilíbrio postural ${ }^{5,6}$. No entanto, devido ao fato de $85 \%$ das informações que o sistema nervoso central recebe do meio externo ocorre por meio as visão ${ }^{7}$, ela torna-se a fonte de entrada principal de informações para a manutenção do controle postural ${ }^{8}$.

Vários estudos correlacionam distúrbios visuais, a exemplo do que ocorre no estrabismo, causadas por doença ou lesão de qualquer uma das composições que formam este complexo sistema (por exemplo: olhos, músculos oculomotores, receptores periféricos) com alterações na função de equilíbrio, função esta que tende a tornar-se insuficiente na presença de comprometimento visual ${ }^{1,9}$.

De acordo com Bricot $^{10}$ pesquisas realizadas na área da neurociência mostram que as mudanças ocorridas no sistema de controle postural dependem em geral de receptores sensitivos internos e externos, onde os olhos e os pés constituem componentes importantes.

Os pés são os elementos base de toda a estrutura corporal humana. É exercida constantemente sobre eles uma enorme pressão, e a eles é dada a função de realizar adaptações em caso de alterações das demais estruturas corporais. O contrário também é verdadeiro, pois Protetti et al. $^{11}$ afirmam que a distribuição de carga no pé reflete no controle postural. Desequilíbrios posturais e alterações biomecânicas podem surgir como consequências da modificação da distribuição da pressão plantar $^{10}$.

A análise dessas pressões desempenha papel importante para uma proposta de prevenção aos transtornos dos membros inferiores, especialmente os do pé, que podem de forma direta interferir no ajuste do controle postural $^{12,13}$.

Há diversas pesquisas que relatam o comprometimento do controle postura em deficientes visuais, porém não há relatos de estudos que avaliem o controle postural, e a pressão plantar, de pessoas que possuam apenas limitação da visão, como é o caso dos indivíduos com alteração da visão. Este estudo teve como objetivo avaliar o controle postural e a pressão plantar em pessoas com alteração visual, utilizando como referência o estrabismo.

\section{METODOLOGIA}

Trata-se de uma pesquisa observacional transversal quantitativa. Para este estudo, compondo o Grupo com Alteração Visual (GAV), foram selecionados 17 indivíduos estrábicos, de faixa etária de 18 a 40 anos, de ambos os gêneros.

Para compor o Grupo Controle (GC), selecionou-se 17 indivíduos com visão normal, de mesma faixa etária, de ambos os gêneros.

Foram incluídos no GAV indivíduos estrábicos, sedentários e com ausência de comprometimento do sistema vestibular, proprioceptivo, auditivo, neurológico e/ou mental.

No GC, participaram indivíduos com visão normal, sedentários e que não apresentaram comprometimento do sistema vestibular, proprioceptivo, auditivo, neurológico e/ou mental.

Foram adotados como critério de exclusão, para ambos os grupos, as características listadas a seguir: indivíduos com presença de comprometimento físico, auditivo, neurológico e/ou mental, portadores de labirintite, praticantes de atividade física, mulheres grávidas e ausência de colaboração nos procedimentos a serem realizados.

A pesquisa foi realizada após aprovação da Comissão de Ética e Pesquisa da Faculdade Centro de Ensino Unificado de Teresina (CEUT), sendo aprovada pelo parecer de protocolo 07634/2013, e por um Comitê de Ética em Pesquisa de uma instituição de ensino local. O estudo obedeceu às normas estabelecidas na Resolução 466/2012 do Conselho Nacional de Saúde. Os voluntários da pesquisa autorizaram a participação na mesma por meio do Termo de Consentimento Livre e Esclarecido.

Para a produção dos dados, todos os indivíduos foram inicialmente submetidos ao exame antropométrico, utilizando-se a balança Filizola $^{\circledR}$ e a fita métrica. Para mensuração da pressão plantar e do equilíbrio utilizou-se a baropodometria computadorizada, uma técnica informatizada que auxilia no diagnóstico de alterações no controle postural e podais, permitindo assim mostrar a relação do pé com a postura durante a posição estática e dinâmica ${ }^{14}$.

A plataforma de força empregada foi a Platforms freeMed ${ }^{\circledR}$, pertencente a Sensor Medica, na qual os resultados foram interpretados pelo software FreeStep ${ }^{\circledR}$. 
Inicialmente foram descritos os procedimentos a serem realizados, bem como a apresentação do equipamento (plataforma baropodometrica), em seguida foi realizado a avaliação da pressão plantar, que consistiu de um procedimento simples, sendo necessário apenas que os participantes ficassem na posição ereta sobre a plataforma com os dois pés, onde foi captado a impressão plantar em apoio bipodal.

Para a avaliação do equilíbrio cada indivíduo foi, então, posicionado em frente à plataforma biomecânica e orientado a: subir na plataforma com os dois pés de forma independente, mantendo pés confortáveis, postura ereta, membros superiores estendidos paralelos ao tronco, cabeça alinhada, mantendose parado e equilibrando-se por 52 segundos, com os olhos abertos. Posteriormente, foi realizado o mesmo procedimento, só que com os olhos fechados. Todos estavam descalços e usando roupas leves durante os procedimentos.

Desta forma, a partir dos dados antropométricos lançados no microcomputador, o software FreeStep ${ }^{\circledR}$ determinou o centro de gravidade de cada sujeito possibilitando a captura da oscilação do mesmo através da plataforma durante a manutenção da postura estática.

Como as amostras apresentaram-se independentes, para a análise dos resultados utilizou-se do teste $t$ de Student e consideraramse significativos os valores em que $p<0,05$. Todos os dados foram expressos em média e desvio padrão (DP).

\section{RESULTADOS}

No que se refere ao exame antropométrico entre os dois grupos não houve diferença estatisticamente significante $(p=0,52)$.

No exame da avaliação da pressão plantar foi demonstrada a mensuração e a comparação da distribuição da pressão nos pontos da região plantar do antepé e retropé. Os parâmetros analisados nessas regiões foram: superfície, carga e relação R/A (Tabela 1).

Tabela 1. Valores médios ( \pm DP) e comparativos do exame da pressão plantar entre os grupos avaliados.

\begin{tabular}{ccccccc}
\hline \multirow{2}{*}{ Variáveis } & \multicolumn{2}{c}{ Esquerda } & \multicolumn{4}{c}{ Direita } \\
\cline { 2 - 7 } & GAV & GC & $\boldsymbol{p}$ & GAV & GC & $\boldsymbol{p}$ \\
\hline Superfície $\left(\mathrm{cm}^{2}\right)$ & & & & & & \\
Antepé & $57,00 \pm 3,58$ & $50,80 \pm 7,57$ & 0,4803 & $55,40 \pm 4,14$ & $51,40 \pm 6,19$ & 0,6060 \\
Retropé & $34,80 \pm 3,39$ & $39,20 \pm 3,53$ & 0,3951 & $35,00 \pm 3,30$ & $38,60 \pm 3,19$ & 0,4554 \\
Carga (\%) & & & & & & \\
Antepé & $22,40 \pm 2,22$ & $27,00 \pm 2,59$ & 0,1589 & $22,40 \pm 2,089$ & $27,20 \pm 2,22$ & 0,1541 \\
Retropé & $20,60 \pm 2,37$ & $27,60 \pm 2,69$ & 0,0873 & $21,40 \pm 2,91$ & $27,40 \pm 2,16$ & 0,1362 \\
Relação R/A (\%) & & & & & & \\
Antepé & $60,60 \pm 4,37$ & $45,60 \pm 4,83$ & 0,0502 & $57,20 \pm 5,54$ & $45,40 \pm 3,82$ & 0,1177 \\
Retropé & $39,40 \pm 4,37$ & $54,40 \pm 4,83$ & 0,0502 & $42,80 \pm 5,54$ & $54,60 \pm 3,82$ & 0,1189 \\
\hline
\end{tabular}

GC - Grupo Controle, GAV - Grupo com Alteração Visual, Teste $t$ de Student não paramétrio (IC $95 \% \mathrm{e}$ significância em $p<0,05)$.

De acordo valor médio e comparativo das variáveis analisadas no exame da pressão plantar observou-se que não houve diferença significativa na distribuição da pressão na região plantar entre os dois grupos.

$\mathrm{Na}$ Tabela 2 estão apresentados os resultados obtidos no exame do equilíbrio postural demonstraram que o deslocamento látero-lateral $(\mathrm{L} / \mathrm{L})$ e a velocidade média de oscilação entre os voluntários investigados, olhos abertos, apresentou diferença estatística significante, onde o GAV em ambas as variáveis apresentou maior oscilação neste sentido, quando comparado com o GC. O deslocamento ântero-posterior $(A / P)$ e a superfície elíptica, não apresentaram diferenças significativas. 
Tabela 2. Comparação da velocidade média ( \pm DP) de oscilações, deslocamento látero-lateral, deslocamento ântero-posterior e superfície elíptica entre os grupos estrábico e normal, com os olhos abertos.

\begin{tabular}{lccc}
\hline \multicolumn{1}{c}{$\begin{array}{c}\text { Variável Estudada } \\
\text { Olhos Abertos }\end{array}$} & GAV & GC & p \\
\cline { 2 - 3 } & $\mathbf{M} \pm$ DP & M \pm DP & \\
\hline $\begin{array}{l}\text { Velocidade Média de } \\
\text { Oscilação (mm/s) }\end{array}$ & $2,90 \pm 1,60$ & $1,10 \pm 0,90$ & 0,0327 \\
Deslocamento L/L (mm/s) & $1,68 \pm 1,43$ & $0,51 \pm 1,51$ & 0,0014 \\
Deslocamento A/P (mm/s) & $0,47 \pm 1,43$ & $0,26 \pm 1,43$ & 0,5968 \\
Superfície Elíptica $\left(\mathrm{mm}^{2}\right)$ & $3,54 \pm 2,93$ & $4,41 \pm 2,90$ & 0,2941
\end{tabular}

GC - Grupo Controle, GAV - Grupo com Alteração Visual, L/L - latero-lateral, A/P - antero-posterior, Teste $t$ de Student não paramétrio (IC $95 \%$ e significância em $p<0,05$ ).

A Tabela 3 mostra que a velocidade média de oscilação, deslocamento L/L e superfície elíptica, olhos fechados, não apresentaram diferença estatística significante. $\mathrm{O}$ deslocamento $A / P$ foi o único que apresentou diferença significante entre os pesquisados nesta condição.

Tabela 3. Comparação da velocidade média ( \pm DP) de oscilações, deslocamento látero-lateral, deslocamento ântero-posterior e superfície elíptica entre os grupos estrábico e normal, com os olhos fechados.

\begin{tabular}{|c|c|c|c|}
\hline \multirow{2}{*}{$\begin{array}{l}\text { Variável Estudada } \\
\text { Olhos Fechados }\end{array}$} & GAV & GC & \multirow{2}{*}{$p$} \\
\hline & $M \pm D P$ & $M \pm D P$ & \\
\hline $\begin{array}{l}\text { Velocidade Média de } \\
\text { Oscilação (mm/s) }\end{array}$ & $1,53 \pm 0,56$ & $1,60 \pm 0,56$ & 0,7266 \\
\hline Deslocamento L/L (mm/s) & $0,35 \pm 1,03$ & $0,54 \pm 1,03$ & 0,5968 \\
\hline Deslocamento A/P (mm/s) & $1,12 \pm 1,29$ & $1,72 \pm 1,59$ & 0,0244 \\
\hline Superfície Elíptica $\left(\mathrm{mm}^{2}\right.$ ) & $6,10 \pm 4,65$ & $6,63 \pm 5,71$ & 0,4562 \\
\hline
\end{tabular}

GC - Grupo Controle, GAV - Grupo com Alteração Visual, L/L - latero-lateral, A/P - antero-posterior, Teste $t$ de Student não paramétrio (IC 95\% e significância em $p<0,05$ ).

\section{DISCUSSÃO}

Os pés são responsáveis pela distribuição da pressão plantar e pela manutenção da postura ortostática. Desta forma tendo os pés como base de suporte, observa-se a sua fundamental importância para o controle postural do indivíduo na realização do movimento e na obtenção do equilíbrio corporal ${ }^{10}$.

Nos resultados obtidos neste estudo não foi encontrada diferença estatisticamente significante na distribuição da pressão plantar dos indivíduos com alteração visual, estrabismo, e com visão normal.

Não há na literatura pesquisas específicas que correlacionem a alteração visual com a distribuição da pressão plantar. Protelli et al. ${ }^{11}$ realizaram uma pesquisa em que avaliaram o tipo de pé, utilizando para isso a distribuição da pressão plantar, em indivíduos com alteração do resíduo visual e com resíduo visual normal, onde os resultados finais mostraram que não há 
diferença estatisticamente significante no tipo de pé entre os dois grupos estudados.

Vários distúrbios visuais, segundo comprovações científicas, estão associados à diminuição do controle postural. A perda de informações visuais, mesmo que parcial, pode tornar o indivíduo mais instáveis, apresentando maior dificuldade em manter-se em equilíbrio, demonstrando, desta forma, comprometimento da coordenação motora e da postura ${ }^{15,16}$.

Como é possível observar na Tabela 3, na análise do equilíbrio no momento olhos aberto, o deslocamento $L / L$ e a velocidade média de oscilação entre os voluntários investigados apresentaram diferença significativa, sendo mais acentuado no GAV.

Muitos autores correlacionam à presença de distúrbios visuais com alterações na função do equilíbrio, o qual tende a tornar-se insuficiente na presença de disfunções do aparelho óptico, originando perturbações no estado de equilíbrio, que pode se manifestar através de desequilíbrios posturais ${ }^{17,18}$. Um comprometimento do sistema de equilíbrio pode reduzir a estabilidade postural, resultando como consequência em aumento da oscilação corporal e/ou alteração da estratégia de movimento ${ }^{1}$. Os resultados encontrados neste estudo mostraram-se congruentes com estas informações.

A maior oscilação corporal no sentido L/L pode está associada a diferentes fatores, entre eles à provável diminuição da base de apoio dos indivíduos com alteração visual, fato este relatado por Kejonen et al. ${ }^{19}$, como meio compensador da falta informação adequado do ambiente por intermédio da visão. Eles relatam que na realização de seus estudos utilizando uma plataforma de força para avaliar o equilíbrio, verificou que a oscilação $\mathrm{L} / \mathrm{L}$ era menor quando os pés eram mantidos afastados.

Przekoracka-Krawczyk et al. ${ }^{20}$ relatam em um estudo, após a avaliação do equilíbrio em indivíduos estrábicos e indivíduos normais utilizando uma plataforma de força, que em geral, os estrábicos mostraram significativamente pior controle do equilíbrio. A diferença entre os dois grupos foi observada principalmente na oscilação lado-a-lado, mas não em excursões ântero-posterior.

Os dados encontrados neste estudo também corroboram com os estudos de Matsuo et al. $^{21}$, que mostraram diminuição do controle de postural, e consequentemente de equilíbrio, em crianças com estrabismo, e Legrand et al. ${ }^{22}$, que relatou baixa estabilidade postural em crianças com estrabismo.

Para o aumento da velocidade média de oscilação observada nos indivíduos voluntários estrábicos, pesquisas anteriores concluíram que a velocidade média de deslocamento (oscilação) é a variável mais sensível para detectar precocemente os distúrbios do equilíbrio relacionados ao sistema óptico ${ }^{23}$.

Na pesquisa de Lions, Bui-Quoc e Bucci ${ }^{24}$ ao comparar o controle postural em crianças estrábicas e crianças não estrábicas, com os dois olhos abertos, verificou-se que a velocidade média de oscilação é significativamente maior em crianças estrábicas quando comparadas a crianças não estrábicas. Esta poderia ser a consequência de um input visual pobre causado pela presença da alteração visual.

Com relação à avaliação do equilíbrio momento olhos fechados, pode-se notar diferença significativa no deslocamento $A / P$, na qual os com visão normal apresentaram maior oscilação, quando comparados com os estrábicos.

De acordo com Mochizuki e Amadio ${ }^{25}$ o equilíbrio é extremamente influenciado pelo sentido da visão e a estabilidade postural tornase mais complicada com os olhos fechados. $\mathrm{Na}$ posição em pé sobre uma plataforma, as informações plantares não são mais as mesmas que sobre o solo firme. Se, nessa situação, fechase os olhos a estabilidade se torna muito mais delicada. A oscilação corporal verificada na manutenção da postura em pé é maior quando a informação visual é eliminada ${ }^{26}$, pois nesta condição o SNC fica dependente das respostas vestibulares e somatossensitivas para detectar potencialmente as oscilações do corpo ${ }^{27,28}$.

A literatura relata que em situações que há diminuição ou privação da acuidade visual ocorre um aumento linear na estabilidade postural, onde se gera uma oscilação anterôposterior proporcionalmente até duas vezes maior que a lateral ${ }^{29}$.

Evidências mostram que a plasticidade cerebral de indivíduos com disfunções visuais permite que áreas comumente associadas com o processamento das informações visuais passem a ser recrutadas para desenvolvimento de outras capacidades $^{30}$. A hipótese de que há estimulação em maior medida dos sentidos remanescentes para manter o controle de equilíbrio ${ }^{31}$. Logo, à presença constante da dificuldade de enxergar, proporciona uma facilidade de adaptação, consequentemente menor oscilação corporal, em 
situações em que ocorra a diminuição ou ausência de informações visuais ${ }^{22}$.

A ausência momentânea dos inputs visuais nos participantes do grupo controle resultou em maior redução no controle de equilíbrio em relação ao grupo experimental na direção $A / P$, o que sugere que os participantes com visão normal não tiveram oportunidade de desenvolver estratégias posturais com base nos inputs aferentes não visuais. Isto se deve ao fato de que quando o indivíduo possui visão normal, confia quase que $100 \%$ a manutenção de equilíbrio corporal sobre os olhos, utilizando muito pouco do sistema vestíbulo-coclear e proprioceptivo $^{32}$. Logo, ao fechar os olhos normalmente ou ao serem vendados, os mecanismos compensatórios entram em ação, entretanto, pelo fato de não serem constantemente exigidos, esta compensação não é eficiente ${ }^{29,33}$.

Corroborando com os nossos resultados, Mayagoitia et al. $^{34}$ ao avaliar a eficácia de métodos para avaliação do equilíbrio em indivíduos com visão normal, nas condições: pés posicionados confortavelmente e olhos abertos (PCOA); pés posicionados confortavelmente e olhos fechados (PCOF); pés juntos e olhos abertos (PCOA), e pés juntos e olhos fechados (PJOF), constataram maior aumento no deslocamento $A / P$ na condição de PCOF. O mesmo ocorreu na condição PJOF.

Przekoracka-Krawczyk et al. ${ }^{20}$ mostrou também entre os resultados de seu estudo, que os indivíduos estrábicos mostraram uma tendência de menor oscilação corporal com os olhos fechados, quando comparado com o grupo dos indivíduos com visão normal, apesar de não apresentar necessariamente diferença significativa.

\section{CONCLUSÃO}

Conclui-se que os indivíduos com alteração visual, estrabismo, avaliadas neste estudo não apresentaram diferença estatisticamente significante na distribuição da pressão plantar ao comparar indivíduos com visão normal. No entanto, foi observado que a alteração visual presente nestes indivíduos parece influenciar negativamente 0 deslocamento látero-lateral e a velocidade média de oscilação na condição olhos aberto. Contudo, na condição olhos fechados eles apresentam menor deslocamento ântero-posterior em relação aos com visão normal.
Sugere-se a realização de outros estudos com maior número de indivíduos avaliados e com outros tipos de alterações visuais para que se analise melhor o impacto das alterações visuais no controle postural e na pressão plantar.

\section{CONFLITOS DE INTERESSES}

Os autores declaram não haver qualquer potencial conflito de interesse que possa interferir na imparcialidade deste trabalho científico.

\section{REFERÊNCIAS}

1. Oliveira, DN, Barreto, RR. Avaliação do equilíbrio estático em deficientes visuais adquiridos. Rev Neurocienc. 2005;13(3):122-7.

2. Loth ED, Rossi AG, Cappellesso PC, Ciena PA. Avaliação da influência do sistema vestibular no equilíbrio de adultos jovens através da posturografia dinâmica foam-laser e plataforma de força. Sem Cienc Biolog Saud. 2008;29(1):5768. DOI: http://dx.doi.org/10.5433/1679$\underline{0367.2008 v 29 n 1 p 57}$

3. Mergner T, Schweigart G, Maurer C, Blümle A. Human postural responses to motion of real and virtual visual environments under different support base conditions. Exp Brain Res. 2005;167(4):535-56. DOI: https://doi.org/10.1007/s00221-005-0065-3

4. Matheron E, Lê TT, Yang Q, Kapoula Z. Effects of a two-diopter vertical prism on posture. Neurosci Lett. 2007;423(3):236-40. DOI: https://doi.org/10.1016/j.neulet.2007.07.016

5. Roth, S. Perioperative visual loss: what do we know, what can we do? $\mathrm{Br} J$ Anaesth. 2009;103(1):31-40.

DOI: http://dx.doi.org/10.1093/bja/aep295

6. Cascio, JC. Somatosensory processing in neurodevelopmental disorders. J Neurodev Disord. 2010;2(2):62-69. DOI: http://dx.doi.org/10.1007/s11689-010-9046-3

7. Friedrich $M$, Grein $H J$, Wicher $C$, Schuetze J, Mueller A, Lauenroth $A$, et al. Influence of pathologic and simulated visual dysfunctions on the postural system. Exp Brain Res. 2008;186(2):305-14. DOI: https://doi.org/10.1007/s00221-007-1233-4 
8. Legrand A, Bui-Quoc E, Bucci MP. Re-alignment of the eyes, with prisms and with eye surgery, affects postural stability differently in children with strabismus. Graefes Arch Clin Exp Ophthalmol. 2012;250(6):849-55. DOI: http://dx.doi.org/10.1007/s00417-011-1845-z

9. Bicas HEA. Estrabismos: da teoria à prática, dos conceitos às suas operacionalizações. Arq Bras Oftalmol. 2009;72(5):585-615. DOI: http://dx.doi.org/10.1590/S0004$\underline{27492009000500002}$

10. Bricot B. Posturologia clínica. São Paulo: CIES Brasil; 2011.

11. Protetti MS, Sankako AN, Carvalho SMRC, Braccialli LMP. Avaliação do controle postural e do tipo do pé de pessoas com deficiência visual. Rev Sob. 2012;13(2):61-6.

12. Menz HB, Morris ME. Clinical determinants of plantar forces and pressures during walking in older people. Gait Posture. 2006;24(2):229-36. DOI: https://doi.org/10.1186/1757-1146-3-11

13. Zammit GV, Menz HB, Munteanu SH. Reliability of the TekScan MatScan(R) system for the measurement of plantar forces and pressures during barefoot level walking in healthy adults. J Foot Ankle Res. 2010;3(11):1-9. DOI: http://dx.doi.org/10.1186/1757-1146-3-11

14. Fortaleza ACS, Martinelli AR, Nozabieli AJL, Mantovani AM, Camargo MR3, Chagas EF, Dalva et al. Avaliação das pressões plantares em diferentes situações por baropodometria. Coll Vitae. 2011;3(1):6-10. DOI: http://dx.doi.org/10.5747/cv.2011.v03.n1.v040

15. Shabana N, Cornilleau-Pérès V, Droulez J, Goh JC, Lee GS, Chew PT. Postural stability in primary open angle glaucoma. Clin Experiment Ophthalmol. 2005;33(3):264-73. DOI: https://doi.org/10.1111/j.1442-

9071.2005.01003.x

16. Sá CG, Bim CR. Análise estabilométrica pré e pós-exercícios fisioterapêuticos em crianças deficientes visuais. Fisiot Mov. 2012;25(4):81119. DOI: http://dx.doi.org/10.1590/S0103$\underline{51502012000400014}$

17. Navarro AS, Fukujima MM, Fontes SV, Matas SLA, Prado GF. Coordenação motora e equilíbrio não são totalmente desenvolvidos em crianças cegas com 07 anos de idade. Arq Neuropsiquiatric. 2004;62(3-A):654-57. DOI: http://dx.doi.org/10.1590/S0004$\underline{282 \times 2004000400016}$

18. Schwesig R, Goldich $Y$, Hahn A, Müller A, Kohen-Raz R, Kluttig A, Morad Y. et al. Postural control in subjects with visual impairment. Eur J Ophthalmol. 2011;21(3):303-9. DOI: http://dx.doi.org/10.5301/EJO.2010.5504

19. Kejonen $\mathrm{P}$, Kauranen $\mathrm{K}$, Vanharanta $\mathrm{H}$. The relationship between anthropometric factors and body-balancing movements in postural balance. Arch Phys Med Rehabil. 2003;84(1):17-22. DOI: https://doi.org/10.1053/apmr.2003.50058

20. Przekoracka-Krawczyk A, Nawrot $P$, Czaińska M, Michalak KP. Impaired body balance control in adults with strabismus. Vision Res. 2014;98:3545.

DOI:

http://dx.doi.org/10.1016/j.visres.2014.03.008

21. Matsuo T, Narita A, Senda $M$, Hasebe $S$, Ohtsuki $\mathrm{H}$. Body sway increases immediately after strabismus surgery. Acta Med Okayama. 2006;60(1):13-24.

22. Legrand A, Quoc EB, Vacher SW, Ribot J, Lebas $\mathrm{N}$, Milleret $\mathrm{C}$, et al. Postural control in children with strabismus: Effect of eye surgery. Neurosci Lett. 2011;501(2):96-101. DOI: http://dx.doi.org/10.1016/j.neulet.2011.06.056

23. Lukman H, Kiat JE, Ganesan A, Chua WL, Khor $\mathrm{KL}$, Choong YF. Strabismus-related prejudice in 56-year-old children. $\mathrm{Br} J$ Ophthalmol. 2010;94(10):1348-51. DOI: http://dx.doi.org/10.1136/bjo.2009.173526

24. Lions C, Bui-Quoc E, Bucci MP. Postural control in strabismic children versus non strabismic age-matched children. Graefes Arch Clin Exp Ophthalmol. 2013;251(9):2219-25. DOI: http://dx.doi.org/10.1007/s00417-013-2372-x

25. Mochizuki, L.; Amadio, A. As informações sensoriais para o controle postural. Fisioter Mov. 2006;19(2):11-8. DOI: http://dx.doi.org/10.1590/S0103$\underline{51502012000400014}$ 
26. Gaertner C, Creux C, Espinasse-Berrod MA, Orssaud C, Dufier JL, Kapoula Z. Benefit of BiOcular Visual Stimulation for Postural Control in Children with Strabismus. PLoS One. 2013;8(4):17. DOI: http://dx.doi.org/10.1371/journal.pone.0060341

27. Horak FB, Wrisley DM, Frank J. The balance evaluation systems test (BESTest) to differentiate balance deficits. Phys Ther. 2009;89(5):484-98. DOI: http://dx.doi.org/10.2522/ptj.20080071

28. Hansson EE, Beckman A, Håkansson A. Effect of vision, proprioception, and the position of the vestibular organ on postural sway. Acta Otolaryngol. 2010;130(12):1358-63. DOI: http://dx.doi.org/10.3109/00016489.2010.49802 $\underline{4}$

29. Sparto PJ, Redfern MS, Jasko JG, Casselbrant $\mathrm{ML}$, Mandel EM, Furman JM. The influence of dynamic visual cues for postural control in children aged 7-12 years. Exp Brain Res. 2006;168(4):505-16. DOI: https://doi.org/10.1007/s00221-005-0109-8

30. Théoret $\mathrm{H}$, Merabet $\mathrm{L}$, Pascual-Leone $\mathrm{A}$. Behavioral and neuroplastic changes in the blind: evidence for functionally relevant cross-modal interactions. J Physiol Paris. 2004;98(1-3):221-33. DOI:

https://doi.org/10.1016/i.jphysparis.2004.03.009

31. Schmid M, Nardone A, De Nunzio AM, Schmid $M$, Schieppati $M$. Equilibrium during static and dynamic tasks in blind subjects: no evidence of cross-modal plasticity. Brain. 2007;130(8):2097107. DOI: https://doi.org/10.1093/brain/awm157

32. Giagazoglou P, Amiridis IG, Zafeiridis A, Thimara M, Kouvelioti V, Kellis E. Static balance control and lower limb strength in blind and sighted women. Eur J Appl Physiol. 2009;107(5):571-9. DOI: http://dx.doi.org/10.1007/s00421-009-1163-x

33. Black AA, Wood JM, Lovie-Kitchin JE, Newman BM. Visual impairment and postural sway among older adults with glaucoma. Optom Vis Sci. 2008;85(6):489-97.

DOI: http://dx.doi.org/10.1097/OPX.0b013e31817882 $\underline{\mathrm{db}}$
34. Mayagoitia RE, Lötters JC, Veltink PH, Hermens $\mathrm{H}$. Standing balance evaluation using a triaxial accelerometer. Gait Posture. 2002;16(1):55-9. DOI: https://doi.org/10.1016/S0966-6362(01)00199-0

Recebido para publicação em 09/12/2015

Revisado em 17/01/2017

Aceito em 19/12/2017 